\title{
Weber-Christian disease: ultrasound can see it
}

\author{
Paolo Agostinis, ${ }^{1}$ Cathryn Anne Scott ${ }^{2}$ \\ ${ }^{1}$ Department of Internal Medicine, Sant'Antonio Abbate Hospital, Tolmezzo (UD); ${ }^{2}$ Pathology, University of Udine, Italy
}

\begin{abstract}
Weber-Christian disease, also known as idiopathic relapsing febrile nodular non-suppurative panniculitis, is a rare inflammatory disorder of the subcutaneous tissue characterized by recurrent nodules often associated with constitutional symptoms, in particular pain and fever. A 50-year-old female patient underwent ultrasound examination of the soft tissues because of relapsing painful subcutaneous nodules of the left arm, accompanied by fever. Ultrasonography showed subcutaneous nodules suggestive of panniculitis. An ultrasound-guided biopsy was performed. The ultrasound-guided biopsy from the nodules with mixed echostructure revealed lobular panniculitis with a mixed cell infiltrate, consistent with Weber-Christian disease, after clinical and pathological exclusion of several differential diagnoses. Recurrent painful subcutaneous nodules associated with fever necessitate a careful medical history and thorough physical examination. Ultrasound can be useful to formulate the hypothesis of panniculitis. Histological examination of a ultrasound-guided biopsy of a nodule with mixed echostructure is needed to confirm the diagnosis. Weber-Christian disease remains a diagnosis of exclusion, when no other cause for the lobular panniculitis can be identified.
\end{abstract}

\section{Introduction}

Weber-Christian disease (WCD) is a rare and poorly understood inflammatory disorder of subcutaneous adipose tissue and was first described in Germany by Pfeifer in $1892 .{ }^{1}$ Its eponym derives from Weber who reported a case of relapsing non-suppurative nodular panniculitis showing phagocytosis of the subcutaneous fat cells by macrophages in $1925^{2}$ and Christian who added the word febrile to this designation in $1928 .^{3}$

Correspondence: Paolo Agostinis, Medicina Interna, Ospedale Sant'Antonio, via Morgagni 18, 33028 Tolmezzo (UD), Italy. Tel.: +39.0433.488.447 - Fax: +39.0433.488.435

E-mail: paoloagostinis.pratocarnico@gmail.com

Key words: Weber-Christian disease, panniculitis, ultrasoundguided biopsy.

Acknowledgments: a special thanks to Dr. Cathryn Anne Scott (Pathology, University of Udine, Italy) for the histological evaluation.

Conflict of interests: the authors declare that they have no potential conflict of interests.

Received for publication: 25 March 2013.

Revision received: 1 August 2013.

Accepted for publication: 9 September 2013.

This work is licensed under a Creative Commons Attribution NonCommercial 3.0 License (CC BY-NC 3.0).

CCopyright P. Agostinis and C.A. Scott, 2014

Licensee PAGEPress, Italy

Italian Journal of Medicine 2014; 8:69-75

doi:10.4081/itjm.2014.170
WCD is characterized by recurrent subcutaneous painful nodules that are often associated with constitutional symptoms such as fever, fatigue, arthralgia, and muscle pain. ${ }^{4}$ In patients with WCD the nodules are typically located at the extremities but may also occur elsewhere: the back, abdominal wall, breasts, face or buttocks. ${ }^{2}$ The lesions are typically tender and initially erythematous; subsequently they ulcerate with serous drainage or serous-bleeding. ${ }^{2}$ Over a period of a few weeks the nodules usually subside but the disease tends to recur at intervals of weeks or months. More unusual cases involve the gastrointestinal tract, heart, lungs, liver, kidneys, mediastinum and the retrobulbar fat with proptosis. ${ }^{4-8}$

The exact etiopathogenesis of WCD is largely unknown. Histological findings are those of a lobular panniculitis with mixed cell infiltrate. In the acute stage of the disease focal necrosis of fat cells with lobular infiltration by neutrophilic granulocytes and lymphocytes is seen; later on macrophages invade the fat lobules and following the inflammatory phase there is replacement of adipose tissue by fibrosis. Rarely there may be associated vasculitis. ${ }^{5,6} \mathrm{WCD}$ is a diagnosis of exclusion, when no other cause for the lobular panniculitis can be identified.

Several treatment options have been described in the literature, mostly in the setting of case reports. The evidence is best for cyclosporine A and corticosteroids. ${ }^{9-17}$ The prognosis is variable but that of lobular panniculitis associated with prominent visceral involvement may be poor. ${ }^{18}$

This report presents the case of a woman with WCD and discusses the signs, symptoms, biochemical profile as well as diagnostic procedures performed and the patient's treatment. 


\section{Case Report}

A 50-year old woman consulted our outpatient clinic because of skin lesions characterized by subcutaneous painful nodules with a serous-bleeding wound (Figure 1) of the left arm. The nodules were recurrent and associated with slight fever, fatigue, arthralgia and myalgia, elevated erythrocyte sedimentation rate and C-reactive protein, as well as mild anemia.

The patient had initially been referred by her physician to a dermatologist. She was diagnosed as having necrotizing suppurative panniculitis by excision biopsy that showed a large area of necrosis with an intense granulocytic component, with features of an abscess (Figure 2). The patient was referred to the Infectious Diseases Clinic and treated with broadspectrum antibiotics (amoxicillin, doxycycline, clindamycin, levofloxacin, teicoplanin) for a long period in order to treat a suspected infection. The lesions did improve temporarily only to reappear. The patient finally consulted our Department of Internal Medicine.

As part of her clinical history the patient reported an episode of erythema nodosum in the past, recurrent oral ulcers and photosensitivity. She did not report any trauma, cold exposure or weight loss. Physical examination showed obesity (body mass index 35), mild pallor and hepatomegaly. Blood investigations showed anemia with normal mean cell volume (hemoglobin $11 \mathrm{~g} / \mathrm{dL}$, normal value $12-16 \mathrm{~g} / \mathrm{dL}$ ), lymphocytopenia $(1220 / \mu \mathrm{L})$ in two controls, and a C-reactive protein level of $35 \mathrm{mg} / \mathrm{L}$ (normal value $0-5 \mathrm{mg} / \mathrm{L}$ ). Leukocyte, eosinophil and platelet counts, creatinine concentration, urine analysis and transaminase levels were within the normal ranges.

Examination of the soft tissues of the left arm using an ultrasound (US) (Esaote Megas Group, Genova, Italy) with a linear $10 \mathrm{MHz}$ probe revealed thickening of the underlying subcutaneous tissue which showed homogeneously increased echogenicity and oval or round fat nodules from 5 to $15 \mathrm{~mm}$ in size located between the skin and muscular fascia. The lumps had sharp outlines and a variable echostructure and echogenicity when compared to the adjacent subcutaneous fat: i) hypo-echogenic with lateral acoustic shadows; ii) hyper-echogenic; and iii) mixed (in part hypo-echogenic and in part echogenic) thus suggesting a diagnosis of panniculitis (Figure 3).

US-guided biopsy was performed using a Menghini 18 gauge needle on the mixed nodules. It revealed lobular panniculitis with a mixed inflammatory cell infiltration (Figure 4). The Gram, Ziehl-Neelsen, Wright and PAS stains and the cultures of the milky aspirate taken from the hypo-echogenic lesions did not show bacterial or fungal micro-organisms. The histological diagnosis of possible WCD was also made by comparing the findings with the previous biopsy.
Radiography of the chest was normal. US and magnetic resonance imaging of the abdomen confirmed mild hepatomegaly with steatosis but without underlying malignancies such as pancreatic carcinoma. Antinuclear antibodies detected by indirect immunofluorescence (IFI on Hep-2 cells) were positive (1:40, speckled pattern) in one control and negative in second one. Antibodies to double-stranded DNA (dsDNA) and antibodies to Smith antigen (Sm) were negative. Antibodies directed against extractable nuclear antigens (ENA) were positive for anti-Ro/SSA. Complement factors $\mathrm{C} 3$ and $\mathrm{C} 4$, creatinine and urine

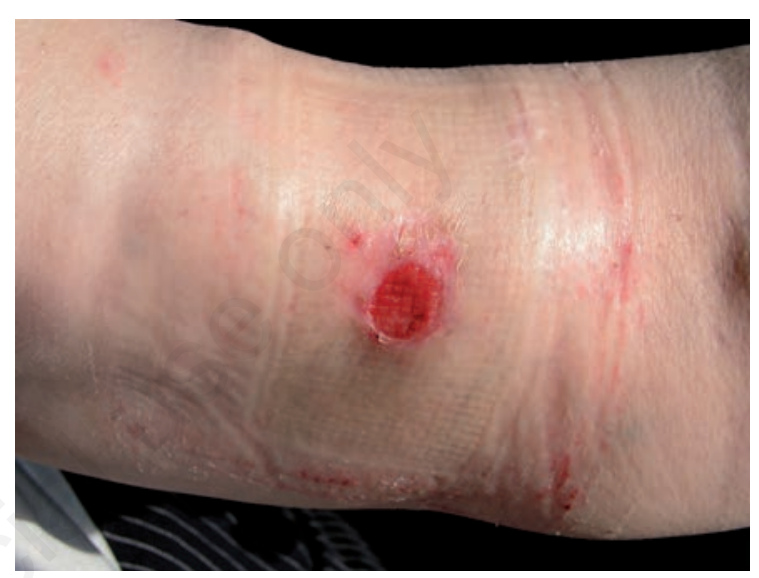

Figure 1. Skin lesion in a 50-year-old woman with Weber-Christian disease. A subcutaneous nodule with an overlying serous-bleeding wound characterizes the lesion on the left arm. On admission of the patient, the skin over and around the lesion showed pattern impressions caused by the dressing and bandaging.

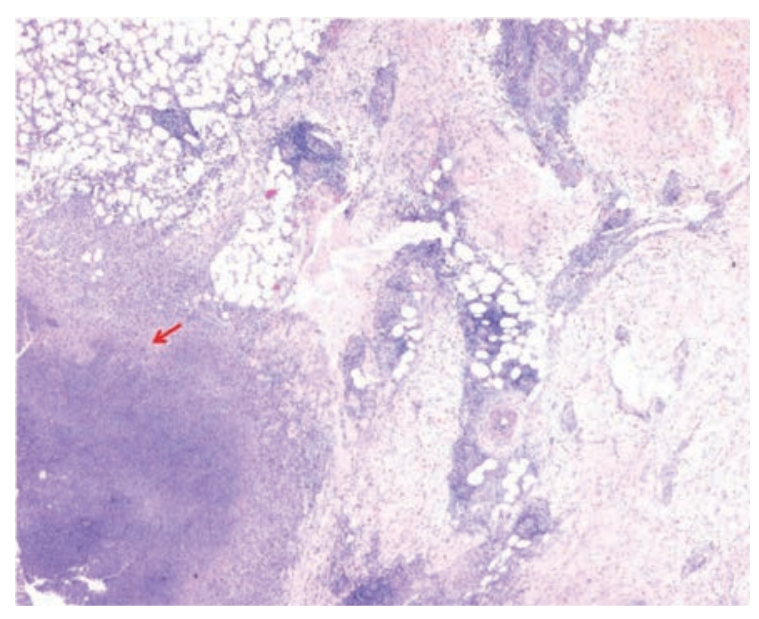

Figure 2. Histology of a nodule on the left arm on a previous excision biopsy carried out without the aid of ultrasound. A large area of necrosis with an intense granulocytic component (arrow) compatible with necrotizing suppurative panniculitis is evident (H\&E-stained 5x). 
analysis, $\alpha 1$-antitrypsin, amylase, lipase, CA 19.9, IgG and IgM anti-cardiolipin antibodies, lupus anticoagulant, antibodies to cyclic citrullinated peptides (anti$\mathrm{CCP}$ ) and antibodies directed against neutrophil cytoplasmic antigens (ANCA) were within the normal range or not detectable, respectively.

A possible diagnosis of WCD associated with borderline criteria for systemic lupus erythematosus (SLE) according to the revised criteria of the American College of Rheumatology for the classification of SLE (Table 1$)^{19}$ was put forward.

The patient was treated with short-term glucocorticoid therapy (prednisone $0.5 \mathrm{mg} / \mathrm{kg} / \mathrm{day}$ ) and longterm hydroxychloroquine treatment $(400 \mathrm{mg} /$ day) and is currently in clinical remission after a follow-up of 8 years (Figure 5 ).

\section{Discussion}

The diagnosis of WCD requires evidence of relapsing fever, systemic inflammation and lobular panniculitis. WCD is a diagnosis of exclusion, when no
Table 1. The revised criteria from the American College of Rheumatology for the classification of systemic lupus erythematosus.

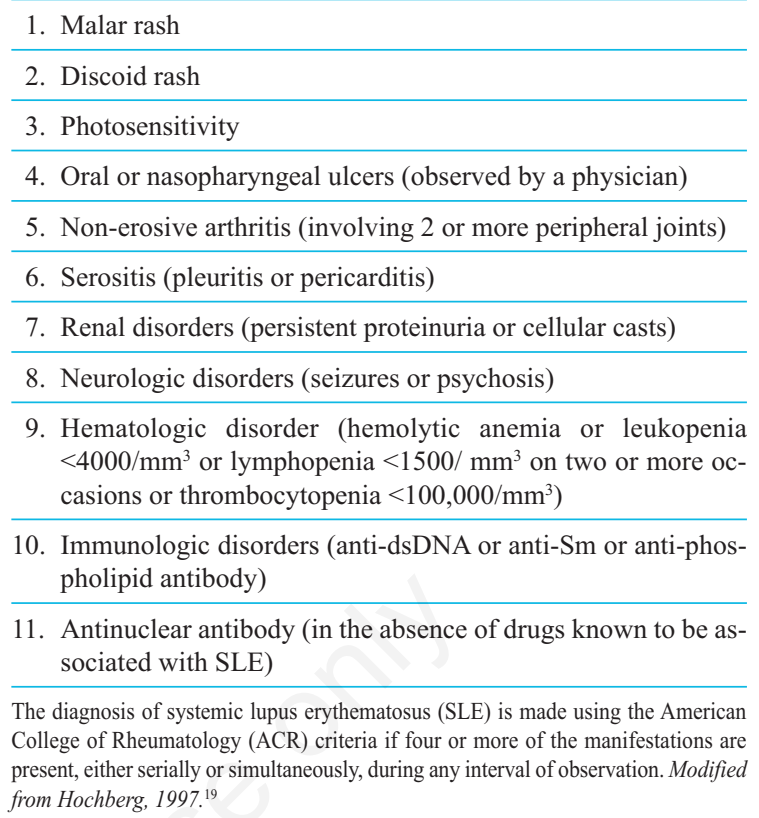
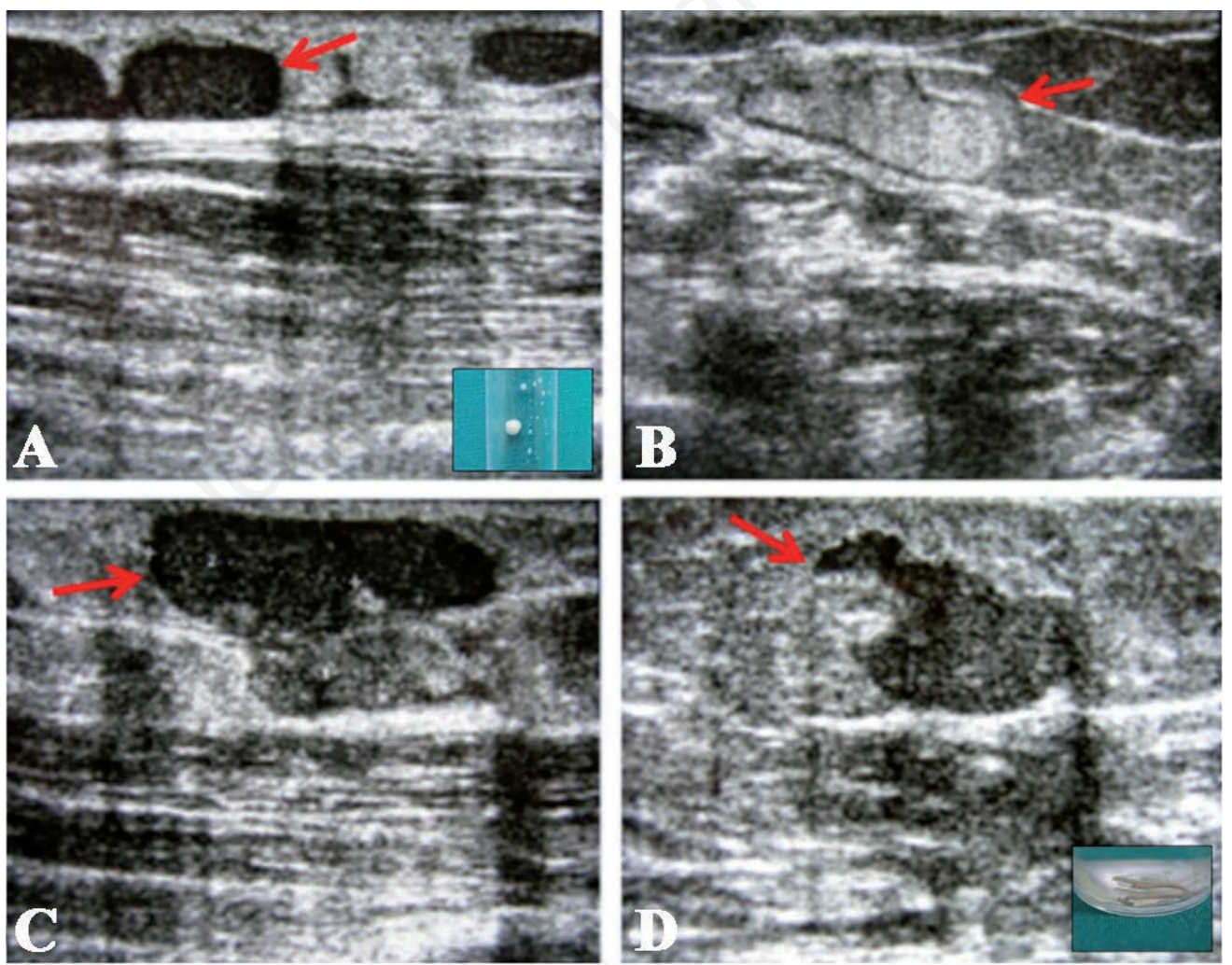

Figure 3. Ultrasound of the skin lesion of the left arm using a linear $10 \mathrm{MHz}$ probe. Oval or round fat nodules from 5 to $15 \mathbf{~ m m}$ in diameter are located between the skin and the muscle fascia. They have sharp outlines and the surrounding subcutaneous tissue, which has homogeneously increased echogenicity, is thickened. The nodules are varied in appearance: hypo-echogenic with lateral acoustic shadows (A), echogenic (B), and mixed, in part hypo-echogenic and in part echogenic when compared to the surrounding subcutaneous fat (C-D). Puncture of the hypo-echogenic nodules (A) showed a milky fluid probably caused by steatonecrosis. 
other cause for the lobular panniculitis can be identified. The most important alternative causes of lobular panniculitis include: SLE, pancreatitis and pancreatic carcinoma, $\alpha 1$-antitrypsin deficiency, rheumatic diseases other than SLE, lymphoma, trauma and Munchausen's syndrome (panniculitis artefacta), certain types of infections and following withdrawal of glucocorticoids (Table 2)..$^{20-27}$

Pancreatitis and pancreatic carcinoma may induce inflammation through the release of pancreatic enzymes that causes fat necrosis (also known as pancreatic or enzymatic panniculitis). Panniculitis in $\alpha 1$-antitrypsin deficiency is thought to result from unopposed proteolysis in the skin possibly related to the formation of $Z$ $\alpha 1$-antitrypsin polymers in the skin. Rheumatic diseases other than SLE that may be associated with lobular panniculitis include complement deficiency, rheumatoid arthritis, vasculitis, myositis, systemic sclerosis, eosinophilic fasciitis, eosinophilia-myalgia syndrome, and anti-cardiolipin antibody syndrome. Hodgkin's and non-Hodgkin's lymphoma, and leukemia may simulate panniculitis. Lobular panniculitis may represent the expression of Munchausen's syndrome (panniculitis artefacta) induced by subcutaneous injection of foreign substances.

Laboratory findings associated with WCD are nonspecific, reflecting acute or chronic inflammation. Patients frequently have an elevated erythrocyte sedimentation rate, anemia, leukocytosis or leukopenia, and occasionally thrombocytopenia. Some patients also have low blood levels of complement factors and circulating immune complexes, consistent with immunologically mediated inflammation.

The clinical suspicion of WCD is usually confirmed by excision biopsy of a nodule. Lobular panniculitis is the essential feature in biopsy specimens. Rarely there may be associated vasculitis. ${ }^{28-30}$

Lesions that may mimic those of WCD (Table 3) include lupus profundus (lupus panniculitis), cutaneous polyarteritis nodosa, erythema nodosum, ery-

Table 2. Conditions associated with lobular panniculitis.

Systemic lupus erythematosus

Pancreatitis

Pancreatic carcinoma

$\alpha 1$-antitrypsin deficiency

Complement deficiency

Rheumatoid arthritis

Vasculitis

Myositis

Systemic sclerosis

Eosinophilic fasciitis

Eosinophilia-myalgia syndrome

Hodgkin's lymphoma and leukemia

Factitial panniculitis

Withdrawal of glucocorticoids
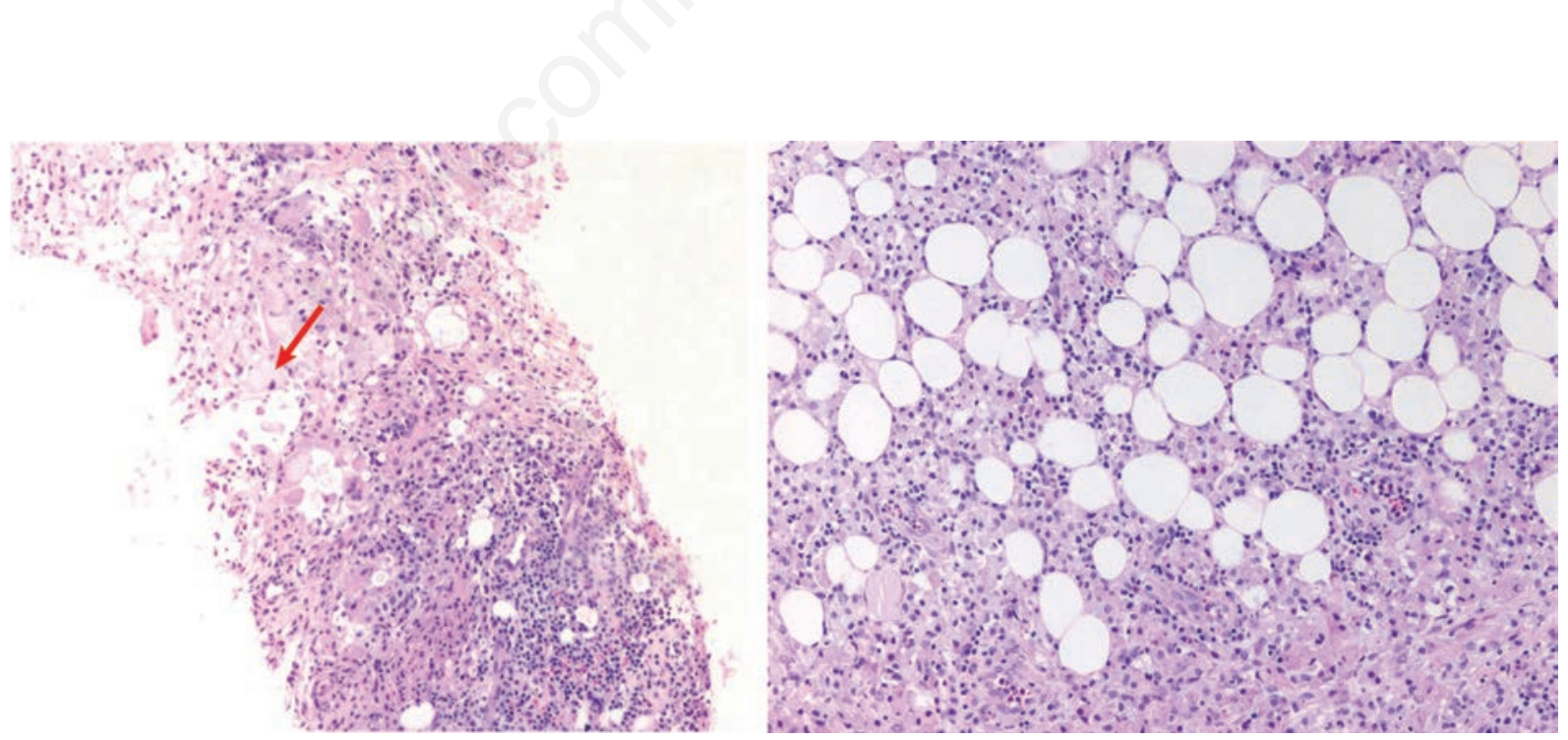

Figure 4. Histology of the nodule with mixed echostructure (left arm, Menghini 18-gauge needle). Two H\&E-stained sections at different magnifications (left 20x, right 40x). The biopsy specimen obtained from a painful, subcutaneous nodule does not include a fully recognizable septum but shows evident lobular panniculitis given the dense infiltration of lymphocytes and histiocytes with occasional perivascular neutrophils. Adipocytes show features of necrosis and foamy degeneration (arrow) (left). At higher magnification (right) various stages of adipocyte degeneration and necrosis are seen together with the dense lympho-histiocytic infiltrate. The small vessels present are lined by activated endothelial cells but no evidence of vasculitis is seen. 
thema induratum, Rothmann-Makai syndrome, and infectious panniculitis.

Lupus panniculitis affects a minority of patients with SLE (2-5\%), and typically develops as erythematous nodules in sites often spared in other panniculitides, including the upper arms, shoulders, face, scalp, and buttocks. It is characterized in particular by hyaline fat necrosis, inflammation predominantly composed of lymphocytes and plasma cells with the presence of lymphoid aggregates and follicle formation, periseptal or lobular lymphocytic panniculitis and lymphocytic vasculitis of small vessels; direct immunofluorescence of the skin is often positive with linear deposition of IgM and $\mathrm{C} 3$ at the dermal-epidermal junction (lupus band test)..$^{20} \mathrm{As}$ in WCD disfiguring lipoatrophy frequently occurs in sites of regressed lesions.

Cutaneous polyarteritis nodosa typically presents clinically as livedo racemosa. Examination of pathology specimens from early lesions reveals neutrophilic vasculitis of arteries and arterioles in the subcutaneous septa associated with dermal leukocytoclastic vasculitis. ${ }^{31}$

Erythema nodosum, the most frequent clinicopathological variant of panniculitis, occurs most often in young women, is usually located in the anterior aspect of the lower legs and resolves spontaneously. In contrast to many other types of panniculitis, ulceration is rare and disfiguring lipoatrophy does not occur in sites of regressed lesions. Histopathological examination reveals a septal panniculitis with a primarily lymphocytic or mixed inflammatory infiltrate and thickened septa usually with no vasculitis. ${ }^{32}$

Erythema induratum usually presents as nodules on the posterior aspect of the lower legs of young women; unlike erythema nodosum, ulceration is common. Although erythema induratum can be idiopathic, it is usually associated with tuberculosis and represents a manifestation of tuberculin hypersensitivity. Histological examination reveals predominantly lobular panniculitis with vasculitis and granulomas. ${ }^{33}$

Rothmann-Makai syndrome is a lobular panniculitis that develops in autoimmune diseases such as juvenile rheumatoid arthritis, diabetes mellitus or Hashimoto's thyroiditis.

Infectious panniculitis usually presents as a lobular

Table 3. Lesions that may mimic Weber-Christian disease.

Lupus profundus (lupus panniculitis)

Cutaneous polyarteritis nodosa

Erythema nodosum

Erythema induratum

Rothmann-Makai syndrome

Infectious panniculitis panniculitis and may be caused by direct inoculation of infectious micro-organisms into the subcutaneous tissue, extension from a nearby focus of infection, or hematogenous dissemination of bacterial, mycobacterial, fungal, protozoan, or viral micro-organisms. ${ }^{34}$

Treatment of WCD depends on the severity of the panniculitis. Antimalarial drugs are suggested in cases with limited cutaneous involvement. ${ }^{35}$ For patients with more severe or extensive skin involvement but no visceral manifestations, various authors have reported a good therapeutic response with corticosteroids. For patients with progressive or disabling disease or with life-threatening visceral panniculitis refractory to treatment with steroids an immunosuppressive regimen including cyclosporine $\mathrm{A}^{9-17}$ and anti-tumor necrosis factor (anti-TFN- $\alpha$ ) is suggested. ${ }^{36,37}$ If there is an identifiable cause of panniculitis, or if there is an associated disease for which specific therapy is indicated, then an attempt to eliminate the cause or treat the underlying disease is an appropriate first step.

The case reported herein presented borderline criteria for the diagnosis of SLE in keeping with the revised criteria of the American College of Rheumatology for the classification of SLE (Table 1).

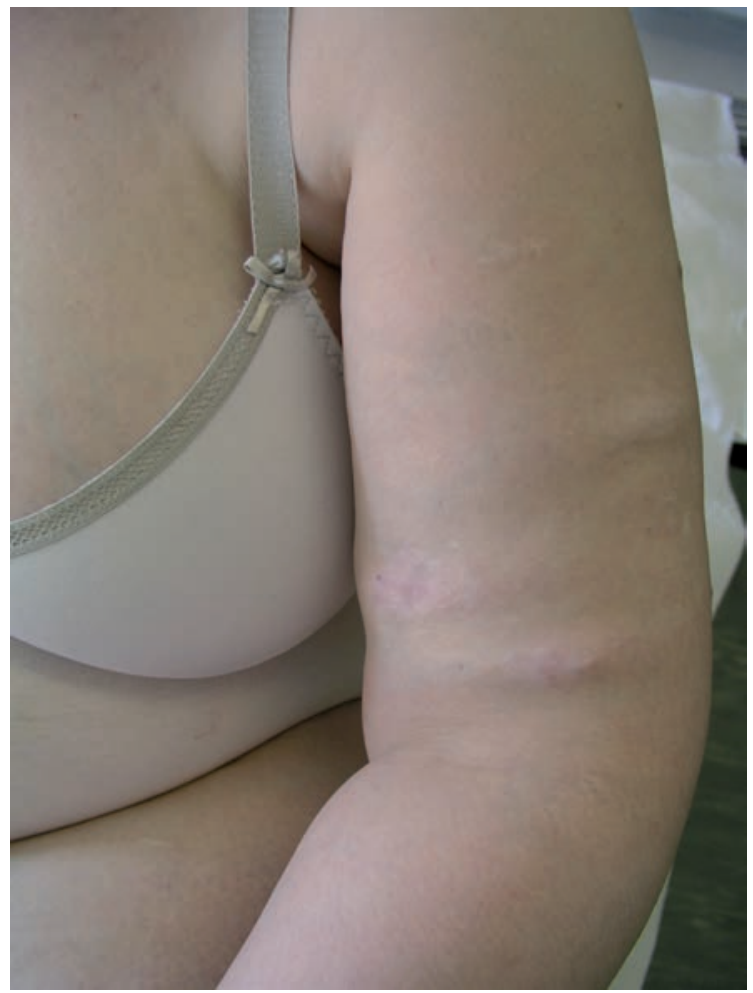

Figure 5. Following treatment with glucocorticoids and hydroxychloroquine, the patient shows scars and irregularity on the surface of the skin caused by replacement of adipose tissue by fibrosis (lipoatrophy). 
The patient had a history of skin rash in reaction to sunlight, painless oral ulcers, and lymphopenia less than $1500 / \mu \mathrm{L}$ on two occasions but a low level of ANA (speckled form), positive only in one control. Anti-dsDNA and anti-Sm antibodies were not detectable and levels of $\mathrm{C} 3$ and $\mathrm{C} 4$ were normal. Anti$\mathrm{Ro} / \mathrm{SSA}$ were present in the absence of xerostomia, xerophthalmia, arthritis, serositis, neurological disorders, proteinuria or cellular casts in urinary sediment, hemolytic anemia or thrombocytopenia.

We could not exclude that this patient had a diagnosis of oligo-SLE associated with panniculitis since some such cases evidence predominantly cytoplasmic antigens and some may be ANA-negative, ${ }^{38}$ furthermore, anti-Ro/SSA are found in approximately 10$60 \%$ of patients associated with both photosensitivity and predominant cutaneous involvement, although the patient, in the long period of follow-up, did not demonstrate further positivity to ANA and ENA or clinical and laboratory features of SLE.

This case represents a good example of the usefulness of US in the study of inflammatory lesions of the subcutaneous tissue. In fact, it enables the true site of the lesions to be determined, thus excluding pathologies that may also involve the dermis, fascia or muscles, it can show whether multiple lesions are all similar or whether they differ from one another and it can be used to guide the appropriate biopsy site thus giving a true idea of the variability of histological features. As already mentioned, the diagnosis of WCD was made after review of the first excision biopsy that showed a true abscess surrounded by damage to the adjacent adipose tissue while the features of foamy degeneration were not seen. It must also be noted that the second biopsy, being single, on its own would probably not have allowed to such a complex diagnosis to have been made given the difficulties inherent in the differential diagnosis of panniculitides. The best system would be to carry out, if possible, more than one biopsy, from different nodules or from areas with differing echogenicity in order to verify the divergent characteristics of these lesions histologically.

\section{Conclusions}

Recurrent painful subcutaneous nodules associated with fever necessitate a careful medical history and physical examination. US can be of great help in the diagnosis of panniculitis allowing selection of the most suitable nodules for biopsy, i.e., the lesions with florid panniculitis rather than the steatonecrotic ones. Compared to excisional biopsy, US-guided needle biopsy has the advantages of being simple, fast and less invasive. WCD remains a diagnosis of exclusion, when no other possible clinical or pathological causes of lobular panniculitis can be identified.

\section{References}

1. Pfeifer V. Uber einen fall von herdweiser atrophic des subcutanin fettgewebes. Deutsches Arch Klin Med 1892;50:438-49.

2. Weber FP, Gray AMA. Chronic relapsing polydermatomyositis with predominant involvement of the subcutaneous fat (panniculitis). Br J Dermatol 1924;36:544.

3. Christian HA. Relapsing febrile nodular non-suppurative panniculitis. Arch Intern Med 1928;42:338-51.

4. Oram S, Cochrane GM. Weber-Christian disease with visceral involvement. An example with hepatic involvement. Br Med J 1958;281-4.

5. White JW JR, Winkelmann RK. Weber-Christian panniculitis: a review of 30 cases with this diagnosis. J Am Acad Dermatol 1998;39:56-62.

6. Niemi KM, Forstrom L, Hannuksela M, et al. Nodules on the legs. A clinical, histological and immunohistological study of 82 patients representing different types of nodular panniculitis. Acta Derm Venereol 1977;57:145-54.

7. Dupont AG, Uerbeelen DL, Six RD. Weber-Christian panniculitis with membranous glomerulonephritis. Am J Med 1983;75:527-34.

8. Wassserman JM, Thung SN, Berman R, et al. Hepatic Weber-Christian disease. Semin Liver Dis 2001;21:115-8.

9. Entzian P, Barth J, Monig H. Treatment of Weber-Christian panniculitis with cyclosporine A. Rheumatol Int 1987;7:181.

10. Usuki K, Kitamura K, Urabe A. Successful treatment of Weber-Christian disease with cyclosporin A. Am J Med 1988;85:276-8.

11. Royle G, Blacklock H, Miller M. Treatment of cytophagic panniculitis with cyclosporin A. Am J Med 1992;92:704-5.

12. Ostroy BE, Athreya BH, Eichenfield AH. Successful treatment of severe cytophagic histiocytic panniculitis with cyclosporine A. Semin Arthritis Rheum 1996;25: 404-13.

13. Viravan S, Wisuthsarewong W, Manonukul J, et al. Successful treatment of cytophagic histiocytic panniculitis by cyclosporin A: a case report. Asian Pac J Allergy Immunol 1997;15:161-6.

14. Iwasaki T, Hamano T, Ogata A. Successful treatment of a patient with febrile, lobular panniculitis (Weber-Christian disease) with oral cyclosporine A: implications for pathogenesis and therapy. Inter Med 1999;38:612-4.

15. Miyasaka N. Steroid-resistant Weber-Christian disease. Intern Med 1999;38:522.

16. Kovacs M, Hafner J, Gabor V. Successful treatment of Weber-Christian panniculitis with cyclosporine A. Orv Hetil 2004;145:827-31.

17. Hinata M, Someya T, Yoshizaki H, et al. Successful treatment of steroid resistant Weber-Christian disease with biliary ductopenia using cyclosporin A. Rheumatology (Oxford) 2005;44:821-3.

18. Panush RS, Yonker RA, Dlesk A, et al. Weber-Christian disease. Analysis of 15 cases and review of the literature. Medicine (Baltimore) 1985;64:181-91.

19. Hochberg MC. Updating the American College of Rheumatology revised criteria for the classification of systemic lupus erythematosus. Arthritis Rheum 1997; 40:1725. 
20. Winkelmann RK. Panniculitis and systemic lupus erythematosus. JAMA 1970;211:472-5.

21. Potts DE, Mass MF, Iseman, MD. Syndrome of pancreatic disease, subcutaneous fat necrosis and polyserositis; a case report and review of literature. Am J Med 1975; 58:417-23.

22. Dahl PR, Su WP, Cullimore KC, et al. Pancreatic panniculitis. J Am Acad Dermatol 1995;33:413-7.

23. García-Romero D, Vanaclocha F. Pancreatic panniculitis. Dermatol Clin 2008;26:465.

24. Breit SM, Clark P, Robinson JP, et al. Familial occurrence of $\alpha 1$-antitrypsin deficiency and Weber-Christian disease. Arch Dermatol 1983;119:198-202.

25. Gross B, Grebe M, Wencker M, et al. New findings in PiZZ $\alpha 1$-antitrypsin deficiency-related panniculitis. Demonstration of skin polymers and high dosing requirements of intravenous augmentation therapy. Dermatology 2009;218:370.

26. Winkelmann RK, Padilha-Goncalves A. Connective tissue panniculitis. Arch Dermatol 1980;116:291-4.

27. Dlesk A, Panush RS. Factitial Weber-Christian disease: a case report. J Rheumatol 1981;8:129-32.

28. Weber FP. Relapsing, non-suppurative, nodular, panniculitis showing phagocytosis of subcutaneous fat cells by macrophages. Br J Dermatol Syph 1925;37:301-11.

29. Spain DM, Foley JM. Non-suppurative, nodular panniculitis (Weber-Christian disease). Am J Pathol 1944;20: 783-7.
30. Forstrom L, Winkelmann, RK. Acute panniculitis; a clinical and histopathological study of 34 cases. Arch Dermatol 1977;113:909-18.

31. Morgan AJ, Schwartz RA. Cutaneous polyarteritis nodosa: a comprehensive review. Int J Dermatol 2010; 49:750-6.

32. Requena L, Yus ES. Erythema nodosum. Dermatol Clin 2008;26:425-38.

33. Mascaró JM Jr, Baselga E. Erythema induratum of bazin. Dermatol Clin 2008;26:439-45.

34. Delgado-Jimenez Y, Fraga J, García-Díez A. Infective panniculitis. Dermatol Clin 2008;26:471-80.

35. Sorensen RU, Abramowsky C, Stern RC. Corticosteroid-sparing effect of hydroxycholoroquine in a patient with early-onset Weber-Christian syndrome. J Am Acad Dermatol 1990;23:1172-4.

36. Al-Niaimi F, Clark C, Thorrat A, Burden AD. Idiopathic lobular panniculitis: remission induced and maintained with infliximab. Br J Dermatol 2009;161:691-2.

37. Mavrikakis I, Georgidias T, FRagiadaki K, et al. Orbital lobular panniculitis in Weber-Christian disease: sustained response to anti-TNF treatment and review of the literature. Surv Ophthalmol 2010;55:584-9.

38. Maddison P, Mogavero H, Provost TT, et al. The clinical significance of autoantibodies to a soluble cytoplasmic antigen in systemic lupus erythematosus and other connective tissue diseases. J Rheumatol 1979;6:189-95. 